\title{
Effect of photodynamic therapy combined with torasemide on the expression of matrix metalloproteinase 2 and sodium-potassium-chloride cotransporter 1 in rat peritumoral edema and glioma
}

\author{
BO LI ${ }^{1}$, CHAO MENG $^{2}$, XUFENG ZHANG ${ }^{3}$, DAMIN CONG $^{1}, \mathrm{XIN} \mathrm{GAO}^{1}$, \\ WANLONG GAO ${ }^{1}$, DONGHUI JU ${ }^{1}$ and SHAOSHAN HU ${ }^{1}$
}

\begin{abstract}
Departments of ${ }^{1}$ Neurosurgery and ${ }^{2}$ Anesthesiology, The Second Affiliated Hospital of Harbin Medical University, Harbin, Heilongjiang 150086; ${ }^{3}$ Department of Internal Medicine-Oncology, The Third Affiliated Hospital of Harbin Medical University, Harbin, Heilongjiang 150081, P.R. China
\end{abstract}

Received February 23, 2015; Accepted January 11, 2016

DOI: $10.3892 / 01.2016 .4210$

\begin{abstract}
Peritumoral edema is a key stage in the infiltration and recurrence of glioma. Photodynamic therapy (PDT) increases the extent of peritumoral edema, which leads to a decrease in the effectiveness of PDT in treating glioma. The present study evaluated the effects of PDT combined with torasemide on the levels of matrix metalloproteinase (MMP) 2 and sodium-potassium-chloride cotransporter (NKCC) 1 in peritumoral edema regions of rat glioma. Adult male Wistar rats were inoculated with rat glioma C6 cells, and the presence of glioma was confirmed using magnetic resonance imaging 7 days subsequent to injection. The rats were randomly assigned to 4 groups $(n=15)$ : Control group, the rats received no treatment; PDT group, the rats received PDT at $80 \mathrm{~J} / \mathrm{cm}^{2}$ for $10 \mathrm{~min}$; torasemide group, the rats received $5 \mathrm{mg} / \mathrm{kg}$ torasemide intraperitoneally; and PDT + torasemide group, the rats received $5 \mathrm{mg} / \mathrm{kg}$ torasemide intraperitoneally for 3 days following PDT at $80 \mathrm{~J} / \mathrm{cm}^{2}$ for $10 \mathrm{~min}$. A total of 5 rats from each group were sacrificed 21 days following injection and the peritumoral edema tissues were harvested. MMP2 and $\mathrm{NKCC} 1$ expression levels were detected in the tissues using immunohistochemistry and western blot analysis. The mRNA expression levels of MMP2 and NKCC1 were observed using reverse transcription-quantitative polymerase chain reaction. Peritumoral edema was measured using a wet-to-dry weight (W/D) ratio, and survival times of the remaining 10 rats in each group were evaluated. Compared with the control group,
\end{abstract}

Correspondence to: Professor Shaoshan Hu, Department of Neurosurgery, The Second Affiliated Hospital of Harbin Medical University, 246 Xuefu Road, Harbin, Heilongjiang 150086, P.R. China

E-mail: shaoshanhu@hotmail.com

Key words: PDT, torasemide, peritumoral edema, MMP2, NKCC1, glioma tumor growth was significantly suppressed in the PDT group and the survival time was prolonged through a reduction in the expression of MMP2 $(\mathrm{P}<0.05)$, and an increased W/D ratio resulted in significantly increased expression of $\mathrm{NKCC} 1$ $(\mathrm{P}<0.05)$. Compared with the PDT group, the expression of $\mathrm{NKCC} 1$ and the W/D ratio in the PDT + torasemide group were significantly decreased $(\mathrm{P}<0.05)$, while no significant difference was observed in the expression levels of MMP2. In conclusion, PDT combined with torasemide prolonged the survival time of rats by inhibiting the growth of glioma through a reduction in the expression of MMP2, and by reducing peritumoral edema through a reduction in the expression levels of NKCC1.

\section{Introduction}

Glioma accounts for 40-60\% of intracranial malignancies (1). The average survival time for individuals with glioma is 12-18 months (2) and the incidence of glioma has increased continuously in the past 20 years (1). Glioma is characterized by invasive growth and an undefined tumor edge; therefore, surgery cannot be used to resect the whole tumor and as a result other treatments are required post-surgery. Currently, adjuvant radiotherapy and chemotherapy are the treatments used post-surgery (3). However, there are severe side effects and complications associated with these treatments, including a high recurrence rate of the tumor (3).

Photodynamic therapy (PDT) refers to a specific wavelength of light that causes photosensitization of cells, which leads to the production of reactive oxygen species that damage cell structures and functions through numerous signaling pathways, and eventually results in the death of cells (4). PDT has been an effective strategy in the treatment of glioma in experimental and clinical studies $(5,6)$. Peritumoral edema is a key stage in the infiltration and recurrence of glioma, and previous studies have demonstrated that PDT increases the permeability of the blood-tumor barrier, which leads to increased peritumoral edema (7). The bilateral effects of PDT 
may lead to the spread of glioma and decrease the effectiveness of PDT in halting the progression of glioma.

Torasemide is a novel loop diuretic that acts on the thick ascending limb of the kidney nephron, inhibits the sodium-potassium-chloride carrier system and increases water excretion (8). The present study evaluated the effects of PDT combined with torasemide on the expression of matrix metalloproteinase (MMP) 2 and sodium-potassium-chloride cotransporter (NKCC) 1 in peritumoral edema of rat glioma.

\section{Materials and methods}

Cell culture. Rat glioma C6 cells were purchased from the Beijing Institutes of Life Science, Chinese Academy of Sciences (Beijing, China) and cultured as monolayers in Gibco ${ }^{\circledR}$ RPMI 1640 medium (Thermo Fisher Scientific, Inc., Waltham, MA, USA) containing Gibco ${ }^{\circledR} 10 \%$ Fetal Bovine Serum (Thermo Fisher Scientific, Inc.) in a humidified incubator (NuAire, Inc., Caerphilly, UK) containing $5 \% \mathrm{CO}_{2}$ at $37^{\circ} \mathrm{C}$. Cells in the exponential phase of growth were used for the present study.

Rat C6 glioma model. All animal protocols in the present study were approved by the Ethics Committee of the Institutional Research Board of Harbin Medical University (Harbin, China; approval no. HMUIRB20150051). Male pathogen-free Wistar rats weighing 220-250 g (60 rats in total; age, 7-8 weeks) were purchased from the Animal Experiment Center of Harbin Medical University, and were kept for $24 \mathrm{~h}$ at room temperature and with free access to water and standard laboratory food prior to C6 cell injection. Injection of C6 cells was performed as previously described (9). Briefly, the rats were anesthetized with $10 \%$ chloral hydrate $(3 \mathrm{ml} / \mathrm{kg}$; CAS no. 302-17-0; Yangzhou Aoxin Chemical Factory, Yangzhou, China) and fixed in stereotaxic apparatus (Motorized Lab Standard Stereotaxic Instrument; catalog no. 51700, Stoelting Co., Wood Dale, IL, USA) for the facilitation of the injection. Following sterilization and a skin incision in the scalp of the rats, a hole was rendered in the skull $1.0 \mathrm{~mm}$ anterior to the anterior fontanel and $1.0 \mathrm{~mm}$ lateral to the sagittal suture. A syringe was inserted $5 \mathrm{~mm}$ into the cerebral cortex and $10 \mu \mathrm{l}$ C6 glioma cell suspension ( $1 \times 10^{6}$ cells) was injected, as described previously (7). The duration of the procedure was $10 \mathrm{~min}$. The syringe remained in the brain for $5 \mathrm{~min}$ followed by a slow extraction. The hole was sealed with bone wax (catalog no. 1029754; B. Braun Melsungen AG; Melsungen, Germany) and the scalp was sutured. Following sterilization, the rats were returned to their accommodation and monitored.

Study design. The presence of glioma was confirmed in the rats 7 days following injection with C6 cells using unenhanced and enhanced magnetic resonance imaging (MRI; Signa HDxt 3.0T; GE Healthcare Bio-Sciences, Pittsburgh, PA, USA), as previously described (10). T1-weighted coronal and axial images were acquired from unenhanced and enhanced scans. Enhanced scans were achieved using an intravenous injection of gadolinium-diethylene triamine pentaacetic acid (Gd-DTPA; 0.4 ml/kg; Magnevist ${ }^{\circledR}$; Bayer AG, Leverkusen, Germany) through the tail vein of the rats. The rats with glioma were randomly assigned to 4 groups $(n=15)$ : Control group, the rats received no treatment; PDT group, the rats received
PDT treatment on day 8 subsequent to injection with C6 cells; torasemide group, the rats received $5 \mathrm{mg} / \mathrm{kg}$ torasemide intraperitoneally (Yoko Pharmaceutical Co., Ltd., Nanjing, China) on day 8 subsequent to injection for a duration of 3 days; and PDT + torasemide group, the rats received $5 \mathrm{mg} / \mathrm{kg}$ torasemide intraperitoneally following PDT on day 8 subsequent to injection for a duration of 3 days. The rats in the control and PDT groups received saline at volumes equivalent to the torasemide received by the rats in the torasemide and PDT + torasemide groups. On day 21 subsequent to injection, 5 rats in each group were sacrificed. Glioma tissues were harvested and processed immediately for wet-to-dry weight (W/D) ratio, histological examination, immunohistochemistry and western blot analysis. The remaining 10 rats in each group were used to determine survival times.

PDT treatment. Hematoporphyrin monomethyl ether (HMME; Shanghai Fudan-Zhangjiang Bio-Pharmaceutical Co., Ltd., Shanghai, China) was administrated via the tail vein of the rats at a dose of $5 \mathrm{mg} / \mathrm{kg}$ on day 8 subsequent to C6 cell injection, and were kept in the dark for $3 \mathrm{~h}$. Subsequently, the rats were anesthetized with $10 \%$ chloral hydrate $(3 \mathrm{ml} / \mathrm{kg})$ and fixed in a stereotaxic apparatus to expand the hole in the skull to $10 \mathrm{~mm}$ in diameter. The tumor was exposed by a microneurosurgical method 5 min later. The optical fiber of the PDT equipment (wavelength, $630 \mathrm{~nm}$; DIOMED 630 PDT Laser; model, T2USA; Diomed Ltd., Cambridge, UK) was placed on the tumor surface and PDT was administered at $80 \mathrm{~J} / \mathrm{cm}^{2}$ in a $30 \mathrm{~mm}^{2}$ region for $10 \mathrm{~min}$, as previously described (8). Following PDT, the hole was sealed with bone wax and the scalp was sutured.

W/D ratio of peritumoral tissues. Regions of peritumoral tissue 1-4 $\mathrm{mm}$ in diameter were resected from glioma. The resected tissues were placed on an ice plate and were immediately weighed. Subsequently, the tissues were desiccated in a $105^{\circ} \mathrm{C}$ oven for $24 \mathrm{~h}$ until a stable dry weight was achieved. The W/D ratio was calculated for water content of the tumor.

Histological observation. Glioma tissues were harvested 21 days following C6 injection and were immediately fixed in 10\% formalin (catalog no. 50-00-0; Dezhou Yun Xin Experimental Instrument Co., Ltd., Dezhou, China), and embedded in paraffin. Tissue sections $6 \mu \mathrm{m}$ thick were cut and stained with hematoxylin and eosin (H\&E; catalog no. C0105, Beyotime Institute of Biotechnology, Shanghai, China) for light microscopy (Eclipse 80i; Nikon Corporation, Tokyo, Japan).

Immunohistochemistry. Paraffinized peritumoral edema tissues were cut into $6-\mu \mathrm{m}$ thick sections for immunohistochemistry. The sections were deparaffinized and hydrated followed by antigen retrieval; the tissues were incubated with $3 \%$ hydrogen peroxide (catalog no. 7722-84-1; Shanghai Ziyi Reagent Factory, Shanghai, China) for $10 \mathrm{~min}$ and subsequently citric acid buffer (catalog no. CW0128; Beijing Tianrui Technology Co., Ltd., Beijing, China) for $3 \mathrm{~min}$. The tissue sections were incubated overnight at $4^{\circ} \mathrm{C}$ with the primary antibodies rabbit anti-rat NKCC-1 antibody (polyclonal; dilution, 1:500; catalog no. ab59791, Abcam, Cambridge, UK), 
rabbit anti-rat MMP2 antibody (polyclonal; dilution, 1:200; catalog no. ab38898; Abcam) and mouse anti-rat glial fibrillary acidic protein antibody (GFAP; polyclonal; dilution, 1:500; catalog no. ab7260; Abcam). Subsequently the tissues were washed with phosphate-buffered saline three times prior to the addition of the goat anti-rabbit secondary antibody (monoclonal; dilution, 1:2,000; catalog no. TA130024; OriGene Technologies, Inc., Beijing, China) for $30 \mathrm{~min}$ at $37^{\circ} \mathrm{C}$. The positive index of a tissue section was the number of cells out of 50 cells that expressed stained cells in 5 fields of view. The tissue sections were blindly examined by a pathologist (Harbin Medical University).

Western blot analysis. Peritumoral edema tissue was ground in liquid nitrogen and dissolved in a RIPA Lysis Buffer (Beyotime Institute of Biotechnology), which contained the protease inhibitor phenylmethanesulfonyl fluoride. The total protein was extracted from the tissue, and the protein concentration was determined using the BCA Protein Assay Kit (catalog no. P0012; Beyotime Institute of Biotechnology) and stored at $-80^{\circ} \mathrm{C}$ for additional analysis. Equal amounts of protein $(>20 \mu \mathrm{g})$ from each group were loaded and resolved using 10\% sodium dodecyl sulfate-polyacrylamide gel electrophoresis and transferred to polyvinylidene difluoride membranes $(0.45 \mu \mathrm{m}$; EMD Millipore, Boston, MA, USA). The membranes were blocked with 5\% non-fat milk in Tris-buffered saline, and subsequently incubated with rabbit anti-rat MMP2 and rabbit anti-rat $\mathrm{NKCC} 1$ antibodies at $4^{\circ} \mathrm{C}$ overnight. The membranes were incubated with goat anti-rabbit secondary antibody (monoclonal; dilution, 1:2,000; catalog no. TA130024; OriGene Technologies, Inc.) for $2 \mathrm{~h}$ at room temperature. The optical density of bands was determined using ImageJ software (version 1.48; National Institutes of Health, Bethesda, MA, USA) and the data were quantified by normalization to the density of $\beta$-actin (OriGene Technologies, Inc.).

Reverse transcription-quantitative polymerase chain reaction (RT-qPCR). Total RNA of each tissue sample was collected using the RNAsimple Total RNA kit (catalog no. DP419; Tiangen Biotech, Co., Ltd., Beijing, China), according to the manufacturer's protocol. A final volume of $20 \mu \mathrm{l}$ cDNA was synthesized from $500 \mathrm{ng}$ total RNA using Super M-MLV Reverse Transcriptase (BioTek China, Beijing, China) and the AffinityScript cDNA Synthesis kit (Agilent Technologies, Inc., Santa Clara, CA, USA), according to the manufacturer's protocol. The qPCR was performed on standardized quantities of cDNA using Exicycler ${ }^{\text {TM }} 96$ (Bioneer Corporation, Daejeon, Korea). The following primers (Shanghai Kehua Bio-engineering Co., Ltd., Shanghai, China) were used: MMP2, forward 5'-GTGGCAATGGAGATGGACA-3' and reverse 5'-GGTCATAATCCTCGGTGGTG-3'; NKCC1, forward 5'-CCTGGGAGAGTTCCACGAT-3' and reverse 5'-TTCGGC AGTGTATGTGACCA- 3 '; and $\beta$-actin, forward 5'-TCAGGT CATCACTATCGGCAAT-3' and reverse 5'-AAAGAAAGG GTGTAAAACGCA-3'. All samples were run in duplicate. Relative mRNA expression levels were calculated as $2^{-\Delta \Delta C q}(11)$.

Statistical analysis. All data were represented as the mean \pm standard deviation. Statistical analyses were performed using the SPSS version 10.0 software (SPSS, Inc., Chicago, IL,
USA). The difference in results between the various groups was analyzed using one-way analysis of variance. Survival data were analyzed by Kaplan-Meier survival analysis, and $\log$ rank test was used to compare the four groups. $\mathrm{P}<0.05$ was considered to indicate a statistically significant difference.

\section{Results}

$H \& E$ staining, GFAP expression and MRI. $\mathrm{H} \& \mathrm{E}$ staining of glioma sections are shown in Fig. 1A. The tissues exhibited invasive growth of glioma and disintegration of the nuclei of the cells. Peritumoral edema tissues exhibited fewer venous lakes compared with normal tissues. The cancer cells invaded normal brain tissues without a defined margin between normal and cancerous brain tissue.

Immunohistochemistry revealed that numerous cells expressed GFAP, which presented as brown granules, and this suggests the presence of glioma (Fig. 1B). A representative MRI scan of a rat with glioma is presented in Fig. 1C. MRI scans were performed 7 days subsequent to injection of C6 cells in all groups to confirm the presence of glioma.

Water content of peritumoral edema tissues. Water content of peritumoral edema tissues was determined by the W/D ratio. The W/D ratio was significantly increased in the PDT group $(5.12 \pm 0.73)$ and decreased in the torasemide group $(4.03 \pm 1.17)$ compared with the control group $(4.76 \pm 0.57)(\mathrm{P}<0.05)$. Compared with PDT group, the W/D ratio decreased significantly in the PDT + torasemide group $(4.79 \pm 0.65 ; \mathrm{P}<0.05)$.

Analysis of MMP2 and NKCC1 expression using immunohistochemistry. The results of MMP2 immunohistochemistry are presented in Fig. 2. There was a decrease in the number of MMP2-positive cells in the PDT group and PDT + torasemide group compared with the control group. However, the number of MMP2-positive cells between the PDT group and PDT + torasemide group did not vary. The number of MMP2-positive cells in the control group and torasemide group also did not vary. Similarly, the positive index in the PDT group (18.3 \pm 1.3$)$ and PDT + torasemide group (16.4 \pm 1.2$)$ was decreased compared to the control group $(30.2 \pm 1.6)$ and torasemide group $(29.4 \pm 0.6)(\mathrm{P}<0.05)$. However, no significant difference was observed in the positive index in the PDT group and PDT + torasemide group.

The results of NKCC1 immunohistochemical analysis are also presented in Fig. 2. Compared with the control group, the number of NKCC1-positive cells was increased in the PDT group and decreased in the torasemide group. Compared with the PDT group, the number of NKCC1-positive cells was decreased in the PDT + torasemide group. Similarly, compared with the control group (30.2 \pm 0.9$)$, the positive index was increased in the PDT group $(32.1 \pm 1.6)$ and significantly decreased in the torasemide group $(23.2 \pm 1.1$; $\mathrm{P}<0.05)$. Compared with the PDT group, the positive index in the PDT + torasemide group was significantly decreased $(25.5 \pm 1.2 ; \mathrm{P}<0.05)$.

Protein expression of MMP2 and NKCC1 using western blot analysis. The protein expression of MMP2 and NKCC1 was determined using western blotting and the results are presented 

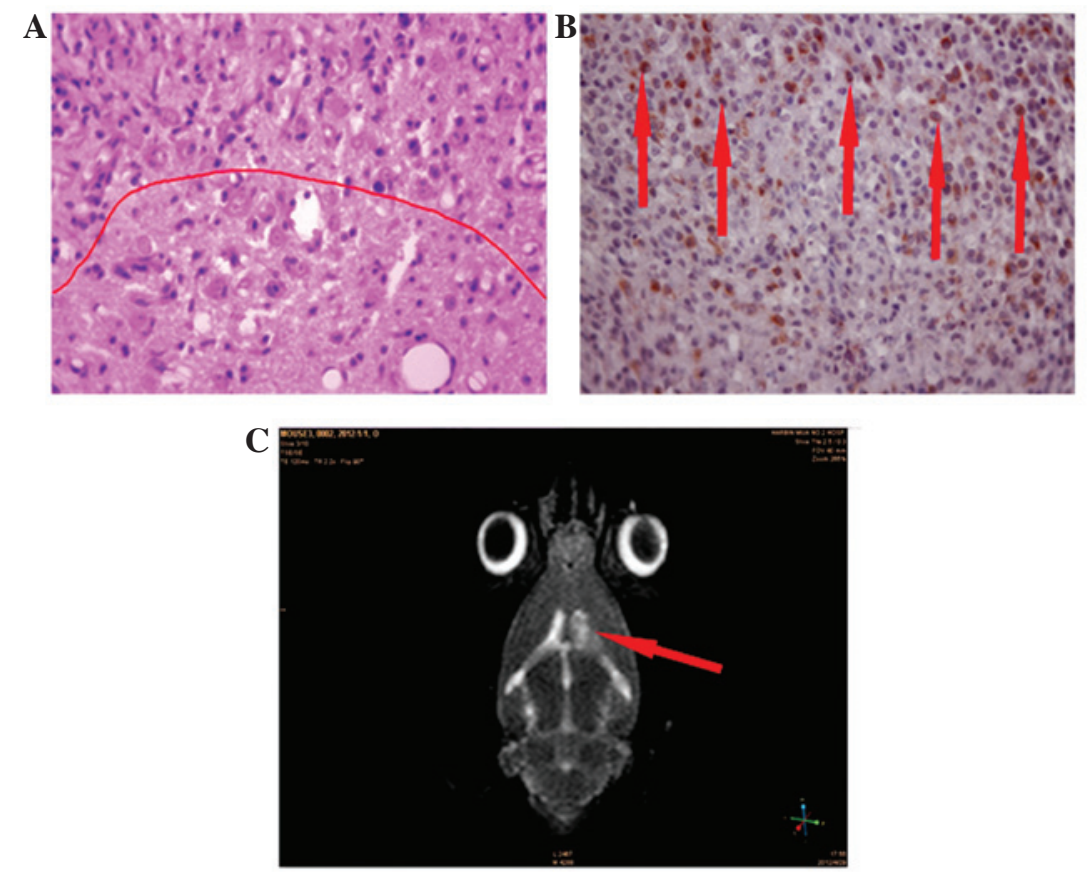

Figure 1. Results of H\&E staining and GFAP expression in the tissues and MRI of a rat 7 days following an injection with rat glioma C6 cells. (A) H\&E staining (magnification, $\mathrm{x} 400$ ). The red line indicates the boundary between cancer and normal brain tissue. (B) GFAP expression as indicated by the red arrows (magnification, $\mathrm{x} 400$. (C) Representative MRI scan. The red arrow indicates the presence of glioma. H\&E, hematoxylin and eosin; GFAP, glial fibrillary acidic protein; MRI, magnetic resonance imaging.
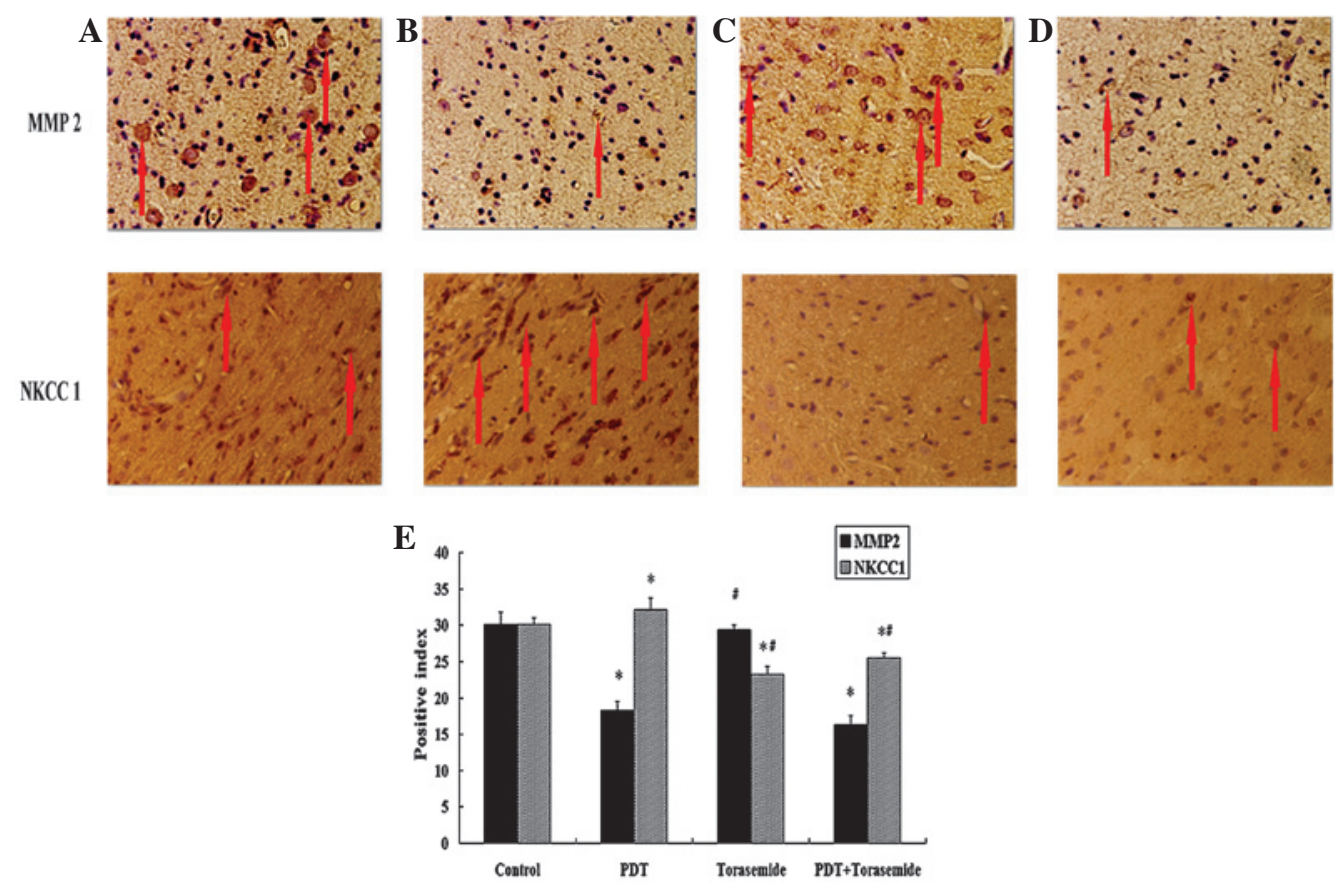

Figure 2. Immunohistochemistry results for the protein expression of MMP2 and NKCC1. (A) Control group; (B) PDT group; (C) torasemide group; and (D) PDT + torasemide group. The red arrows indicate the protein-positive cells; magnification, $\mathrm{x} 400$. (E) The positive expression index for all the groups. Data are shown as the mean \pm standard deviation. ${ }^{*} \mathrm{P}<0.05$ vs. control group; ${ }^{\sharp} \mathrm{P}<0.05$ vs. PDT group. PDT, photodynamic therapy; MMP2, matrix metalloproteinase 2; NKCC1, sodium-potassium-chloride cotransporter 1.

as the ratio of optical density to $\beta$-actin (control). The protein expression of MMP2 was decreased in the PDT group and PDT + torasemide group compared with the control group (Fig. 3; P<0.05). The protein expression of MMP2 between the PDT and PDT + torasemide group had no significant difference. The protein expression of NKCC1 in the PDT group was significantly increased compared with the control group, and the protein expression of NKCC1 in the torasemide group was significantly decreased compared with the control group (Fig. 3; $\mathrm{P}<0.05$ ). The NKCC1 protein expression 

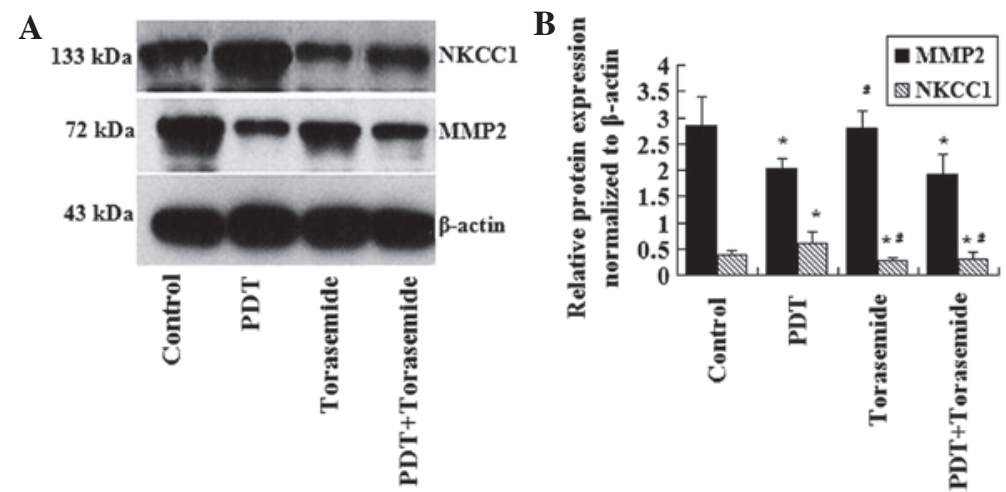

Figure 3. Protein expression of MMP2 and NKCC1 determined using western blot analysis. (A) Representative bands of MMP2, NKCC1 and $\beta$-actin. (B) Relative expression of MMP2 and NKCC1 normalized to $\beta$-actin. Data are shown as the mean \pm standard deviation. ${ }^{*} \mathrm{P}<0.05$ vs. control group; ${ }^{*} \mathrm{P}<0.05$ vs. $\mathrm{PDT}$ group. PDT, photodynamic therapy; MMP2, matrix metalloproteinase 2; NKCC1, sodium-potassium-chloride cotransporter 1.

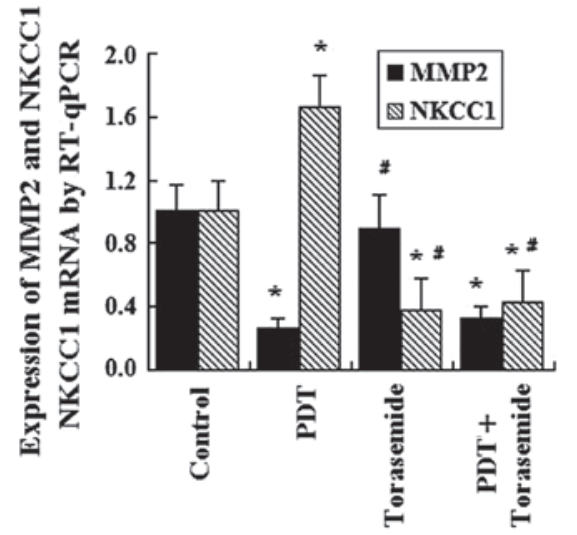

Figure 4. mRNA expression of MMP2 and NKCC1 was observed using RT-qPCR. Data are shown as the mean \pm standard deviation. * $\mathrm{P}<0.05$ vs. control group; "P<0.05 vs. PDT group. PDT, photodynamic therapy; MMP2, matrix metalloproteinase 2; NKCC1, sodium-potassium-chloride cotransporter 1; RT-qPCR, reverse transcription-quantitative polymerase chain reaction.

in the PDT + torasemide group was significantly decreased compared with the PDT group $(\mathrm{P}<0.05)$.

Expression of MMP2 and NKCC1 mRNA using RT-qPCR. The mRNA expression of MMP2 and NKCC1 in the peritumoral edema tissues was determined using RT-qPCR (Fig. 4). The mRNA expression levels of MMP2 in the PDT group and PDT + torasemide group were decreased compared with the control group $(\mathrm{P}<0.05)$. A statistical diference was not observed between the mRNA expression levels of MMP2 in the PDT group and PDT + torasemide group. The mRNA expression levels of NKCC1 in the PDT group increased significantly compared with the control group, and the value in the torasemide group decreased significantly compared with the control group $(\mathrm{P}<0.05)$. Compared with the PDT group, the mRNA expression levels of NKCC1 in the PDT + torasemide group were significantly decreased $(\mathrm{P}<0.05)$.

Survival time. Survival time was analyzed using Kaplan-Meier analysis. The mean survival times for the PDT and torasemide groups were $30.6 \pm 2.4$ and $28.3 \pm 2.0$ days, respectively, which were significantly increased compared with the control

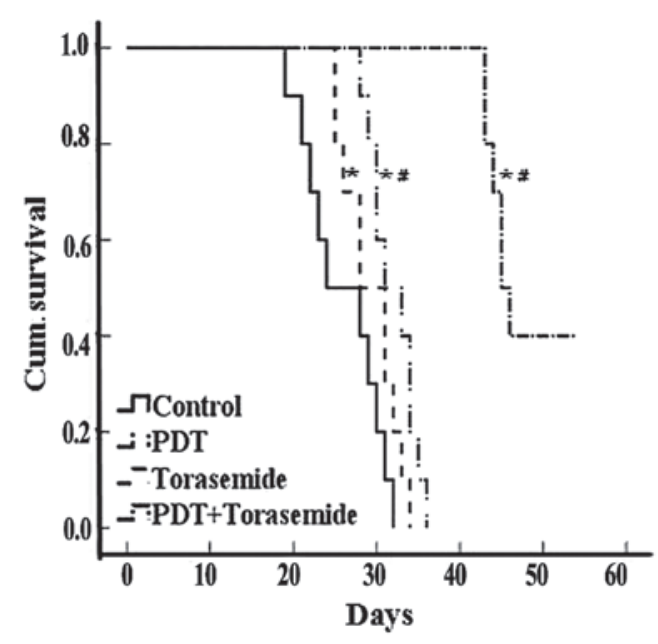

Figure 5. Kaplan-Meier survival time curves for the control, PDT, torasemide and PDT + torasemide treated groups. ${ }^{*} \mathrm{P}<0.05$ vs. control group; ${ }^{\#} \mathrm{P}<0.05$ vs. PDT group. PDT, photodynamic therapy.

group $(24.5 \pm 2.2)(\mathrm{P}<0.05)$. The mean survival time in the PDT + torasemide group was $47.7 \pm 2.5$ days, which was significantly increased compared with the PDT and torasemide groups (Fig. 5; $\mathrm{P}<0.05$ ).

\section{Discussion}

Peritumoral edema is located between the gray, soft glioma tissue and the white, moist normal tissue, which is relatively easy to identify under a light microscope (6). Peritumoral edema contains glioma cells and is a key stage during the invasive growth and recurrence of glioma. A previous study demonstrated that PDT treatment leads to increased permeability of the blood-tumor barrier, which increases the extent of peritumoral edema (12). However, whether the invasion ability of glioma cells is caused by a water and salt metabolic imbalance requires investigation. Torasemide is a novel loop diuretic (13), and has a role in the medullary thick ascending limb of the kidney where it inhibits the sodium-potassium-chloride carrier system, which leads to an increase in the excretion of sodium, potassium, chloride and water in the urine that may in theory reduce peritumoral edema. 
Currently, the mechanism that causes peritumoral edema is unknown; however, the invasion abilities and mechanisms that are associated with cerebral edema may be involved (14), and the invasion of glioma is considered to be an important factor $(14,15)$. Gene products produced as a result of cerebral edema have been demonstrated to transport tumor cells, water, macromolecules and plasma through the basement membranes of vessel walls to the peritumoral region, which leads to the development of peritumoral edema and glioma invasion; this process is closely associated with the MMP family (16-18). The growth of malignant glioma compresses normal tissue and releases a large number of neurotransmitters, which alters the environment of the tissue to provide beneficial anatomical and physicochemical alterations that increase the growth of glioma.

MMPs are secreted by connective tissue cells, macrophages and certain tumor cells and degrade extracellular matrix proteolytic enzymes associated with gliomas in a zinc-dependent manner (17). MMP2 and peritumoral edema have been demonstrated to be increased with a more severe glioma grade (6). The degradation of the extracellular matrix and basement membrane is a result of an imbalance between MMPs and tissue inhibitors of MMPs (19). An increased expression of MMP2 in glioma degrades the extracellular matrix and basement membrane of blood vessels directly and indirectly, which leads to the invasion of glioma and the development of peritumoral edema. NKCC1 induces sodium, chloride and potassium ions to enter a cell $(20,21)$, while the potassium and chlorine cotransporter (KCC2) induces chloride and potassium ions to leave a cell. The balance between $\mathrm{NKCC} 1$ and $\mathrm{KCC} 2$ mediates the electrochemical gradient of the membrane $(22,23)$. NKCC1 has an increased expression in peritumoral edema, which leads to an imbalance in ions that causes the cell to swell and results in cell dysfunction (22). Therefore, vasogenic edema and cytotoxicity of cells coexist in peritumoral edema.

PDT uses $630 \mathrm{~nm}$ wavelengths of visible light to cause photosensitization of cells, which aggregates tumor tissue and eventually leads to tumor cell death (24). HMME is a novel stable photosensitizer with a high specificity to tumor tissue, and has been used clinically for numerous years (25). The present study demonstrated that HMME-PDT is a useful method to induce tumor cell apoptosis and necrosis effectively. Cell apoptosis induced by PDT may be caused by the release of a variety of mitochondrial apoptotic factors, including cytochrome $\mathrm{C}$, apoptosis inducing factor and procaspases that activate caspase-3 (25). The present results demonstrate that PDT decreased the protein and mRNA expression of MMP2, but increased the protein and mRNA expression of $\mathrm{NKCC1}$, which may affect the effectiveness of PDT. In addition, PDT increased the water content of peritumoral edema tissues and destroyed blood vessels, which was confirmed by the development of ischemia and hypoxia in the peritumoral edema region, and aggravated ischemia and hypoxia of peritumoral tissue. This may cause high levels of intracellular chloride due to an increased expression of NKCC1.

Torasemide causes an increase in the excretion of sodium, potassium, chloride and water in the urine. It has a long half-life and a high plasma protein binding and bioavailability rate. Torasemide inhibits $\mathrm{NKCC} 1$ in peritumoral edema tissue, and is located at the cancerous tissue boundary. It may then relocate to the blood due to the osmotic pressure gradient, which leads to an attenuation of peritumoral edema (26). In the present study, torasemide inhibited the protein and mRNA expression of $\mathrm{NKCC1}$ and decreased the W/D ratio. This suggests that torasemide affects NKCC1 directly and indirectly and decreases peritumoral edema.

In the present study, torasemide treatment following PDT decreased the protein and mRNA expression of NKCC1 and $\mathrm{W} / \mathrm{D}$ ratio. There was no significant increase in the protein and mRNA expression of MMP2. In addition, torasemide treatment following PDT greatly increased the survival time of rats with glioma.

In conclusion, PDT combined with torasemide treatment significantly prolonged the survival time of rats with glioma, and reduced the invasion of glioma and peritumoral edema. PDT combined with torasemide may be more effective if it affects MMP2 and NKCC1; however, even though this treatment strategy did not affect MMP2 it may be a beneficial treatment strategy for patients with glioma.

\section{References}

1. Davis FG, Kupelian V, Freels S, McCarthy B and Surawicz T: Prevalence estimates for primary brain tumors in the United States by behavior and major histology groups. Neuro Oncol 3: 152-158, 2001.

2. Li JH, Song DY, Xu YG, Huang Z and Yue W: In vitro study of haematoporphyrin monomethyl ether-mediated sonodynamic effects on C6 glioma cells. Neurol Sci 29: 229-235, 2008.

3. Chibbaro S, Benvenuti L, Caprio A, Carnesecchi S, Pulerà F, Faggionato F, Serino D, Galli C, Andreuccetti M, Buxton N and Gagliardi R: Temozolomide as first-line agent in treating high-grade gliomas: Phase II study. J Neurooncol 67: 77-81, 2004.

4. Choudhary S, Nouri K and Elsaie ML: Photodynamic therapy in dermatology: A review. Lasers Med Sci 24: 971-980, 2009.

5. Tsutsumi M, Miki Y, Akimoto J, Haraoka J, Aizawa K, Hirano K and Beppu M: Photodynamic therapy with talaporfin sodium induces dose-dependent apoptotic cell death in human glioma cell lines. Photodiagn Photodyn Ther 10: 103-110, 2013.

6. Zhan Q, Yue W and Hu S: Effect of photodynamic therapy and endostatin on human glioma xenografts in nude mice. Photodiagn Photodyn Ther 8: 314-320, 2011.

7. Zhang X, Li X and Wu J: Experimental study on the invasion of glioma in vivo. Zhonghua Yi Xue Za Zhi 81: 150-153, 2001 (In Chinese).

8. Schreiber S, Gross S,Brandis A,Harmelin A, Rosenbach-Belkin V, Scherz A and Salomon Y: Local photodynamic therapy (PDT) of rat C6 glioma xenografts with Pd-bacteriopheophorbide leads to decreased metastases and increase of animal cure compared with surgery. Int J Cancer 99: 279-285, 2002.

9. Zelenkov P, Baumgartner R, Bise K, Heide M, Meier R, Stocker S, Sroka R, Goldbrunner R and Stummer W: Acute morphological sequelae of photodynamic therapy with 5-aminolevulinic acid in the C6 spheroid model. J Neurooncol 82: 49-60, 2007.

10. Ulmer S, Reeh M, Krause J, Herdegen T, Heldt-Feindt J, Jansen O and Rohr A: Dynamic contrast-enhanced susceptibility-weighted perfusion MRI (DSC-MRI) in a glioma model of the rat brain using a conventional receive-only surface coil with a inner diameter of $47 \mathrm{~mm}$ at a clinical $1.5 \mathrm{~T}$ scanner. J Neurosci Methods 172: 168-172, 2008.

11. Livak KJ and Schmittgen TD: Analysis of relative gene expression data using real-time quantitative PCR and the 2(-Delta Delta C(T)) Method. Methods 25: 402-408, 2001.

12. Zhang X, Cong D, Shen D, Gao X, Chen L and Hu S: The effect of bumetanide on photodynamic therapy-induced peri-tumor edema of C6 glioma xenografts. Lasers Surg Med 46: 422-430, 2014.

13. Fowler SF and Murray KM: Torsemide: A new loop diuretic. Am J Health Syst Pharm 52: 1771-1780, quiz 1814-1815, 1995.

14. Nase G, Helm PJ, Enger R and Ottersen OP: Water entry into astrocytes during brain edema formation. Glia 56: 895-902, 2008. 
15. Kiyatkin EA, Brown PL and Sharma HS: Brain edema and breakdown of the blood-brain barrier during methamphetamine intoxication: Critical role of brain hyperthermia. Eur J Neurosci 26: 1242-1253, 2007.

16. Scott JG, Bauchet L, Fraum TJ, Nayak L, Cooper AR, Chao ST, Suh JH, Vogelbaum MA, Peereboom DM, Zouaoui S, et al: Recursive partitioning analysis of prognostic factors for glioblastoma patients aged 70 years or older. Cancer 118: 5595-5600, 2012.

17. Lebeau A, Müller-Aufdemkamp C, Allmacher C, Sauer U, Nerlich A, Lichtinghagen $R$ and Löhrs U: Cellular protein and mRNA expression patterns of matrix metalloproteinases-2, -3 and -9 in human breast cancer: Correlation with tumour growth. J Mol Histol 35: 443-455, 2004.

18. VanMeter TE, Rooprai HK, Kibble MM, Fillmore HL, Broaddus WC and Pilkington GJ: The role of matrix metalloproteinase genes in glioma invasion: Co-dependent and interactive proteolysis. J Neurooncol 53: 213-235, 2001.

19. Nelson AR, Fingleton B, Rothenberg ML and Matrisian LM: Matrix metalloproteinases: Biologic activity and clinical implications. J Clin Oncol 18: 1135-1149, 2000.

20. Haas $\mathrm{M}$ and Forbush B III: The $\mathrm{Na}-\mathrm{K}-\mathrm{Cl}$ cotransporters. J Bioenerg Biomembr 30: 161-172, 1998.
21. Kaplan MR, Mount DB, Delpire E, Gamba G and Hebert SC: Molecular mechanisms of $\mathrm{NaCl}$ cotransport. Annu Rev Physiol 58: 649-668, 1996.

22. Hartmann AM, Blaesse P, Kranz T, Wenz M, Schindler J, Kaila K, Friauf E and Nothwang HG: Opposite effect of membrane raft perturbation on transport activity of $\mathrm{KCC} 2$ and NKCC1. J Neurochem 111: 321-331, 2009.

23. Lang F, Ritter M, Gamper N, Huber S, Fillon S, Tanneur V, Lepple-Wienhues A, Szabo I and Gulbins E: Cell volume in the regulation of cell proliferation and apoptotic cell death. Cell Physiol Biochem 10: 417-428, 2000.

24. Li JH, Chen ZQ, Huang Z, Zhan Q, Ren FB, Liu JY, Yue W and Wang Z: In vitro study of low intensity ultrasound combined with different doses of PDT: Effects on C6 glioma cells. Oncol Lett 5: 702-706, 2013.

25. Devi DG, Cibin TR and Abraham A: Bis (3,5-diiodo-2,4,6-trihydroxyphenyl) squaraine photodynamic therapy induces in vivo tumor ablation by triggering cytochrome $\mathrm{c}$ dependent mitochondria mediated apoptosis. Photodiagn Photodyn Ther 10: 510-517, 2013.

26. Kahle KT, Staley KJ, Nahed BV, Gamba G, Hebert SC, Lifton RP and Mount DB: Roles of the cation-chloride cotransporters in neurological disease. Nat Clin Pract Neurol 4: 490-503, 2008. 